thicker among RA patients with rheumatoid factor (RF) positive ( $p=0.015$, $\mathrm{Cl}$ :0.53-0.66). LAD coronary artery wall thickness was increased in early RA compare to controls $(0.61 \pm 0.04 \mathrm{~mm}(\mathrm{Cl}: 0.52-0.70)$; and $0.48 \pm 0.08 \mathrm{~mm}(\mathrm{Cl}: 0.44-$ 0.51 ) respectively, $\mathrm{p}=0.001$

Conclusions: Early RA patients have increased coronary arteries atherosclerotic burden compared to controls matched for age, and sex. Rheumatoid factor positivity, high Disease Activity Score and Patient Global Assessment of disease activity were associated with coronary wall thickness.

Disclosure of Interest: None declared

DOI: 10.1136/annrheumdis-2018-eular.4254

\section{AB0403 FACTORS CONTRIBUTE TO THE LEVEL URIC ACID IN RHEUMATOID ARTHRITIS}

S. Hannawi ${ }^{1}$, I. Al Salmi ${ }^{2}$, H. Hannawi ${ }^{3} .{ }^{1}$ Rheumatology, Ministry of Health and Prevention of United Arab Emirates, Dubai, United Arab Emirates; ${ }^{2}$ The Royal Hospital, Muscat, Oman; ${ }^{3}$ Ministry of Health and Prevention of United Arab Emirates, Dubai, United Arab Emirates

Background: Uric acid (UA) is a strong correlate of renal dysfunction in rheumatoid arthritis (RA), even in the absence of crystal deposition, by causing endothelial dysfunction, intrarenal vascular disease and renal impairment. In hyper or normo-uricaemia RA, UA was the strongest independent predictor of GFR, even after adjustments for most of the potential confounding factors. Additionally, in RA patients UA had been found to be an independent predictor of hypertension and cardiovascular disease (CVD).

Objectives: this study aimed to investigate what could be the determinants of the UA level in RA patients.

Methods: RA patients with no clinically evident gout, CVD, thyroid disease, liver or renal disease were studied. Serum UA was obtained for all the patients. Renal function parameters, RA disease characteristics, inflammatory markers, and traditional CVD risk factors relation to uric acid level was examined using simple linear regression analysis. To test for the independence association between uri acid and the variables of interest, multiple model was built for the same dependent and independents variables. Statistical significance was accepted at $p$-value $<0.05$. Results: The study recruited 86 consecutive patients meeting the 1987 RA revised ACR criteria, attending routine outpatient clinics at the Department of Rheumatology. Basic demographics and clinical characteristics of were obtained. Of the total 86 patients, $10(11 \%)$ were men and $77(89 \%)$ were women. The mean age of the participants was $47 \pm 14$ years, with mean RA duration of $68 \pm 87$ months. 64 out of 86 participants (74\%) had rheumatoid factor positive.

The mean UA value was $255 \pm 86$ umol/L (NR: 180-340). The mean GFR, calculated using modified MDRD (Modification of Diet in Renal Disease) formula was $133 \pm 52 \mathrm{ml} / \mathrm{min} / 1.73 \mathrm{~m}^{2}$

Using univariate analysis revealed a positive linear relationship between uric acid level and each of the age of the participants ( $p<0.016, \mathrm{Cl}: 0.31,2.92)$, age at $\mathrm{RA}$ symptoms onset $(p<0.04, \mathrm{Cl}$ : $0.025,0.039)$, age at RA diagnosis $(p<0.03, \mathrm{Cl}$ : $0.101,2.565)$, systolic blood pressure ( $p=0.04, \mathrm{Cl}: 0.054,2.167)$, diastolic blood pressure (DBP) $(p=0.02, \mathrm{Cl}: 0.322,3.777)$, monocytes absolute count $(\mathrm{p}=0.014$, $\mathrm{Cl}: 2.510,4.801)$, monocytes percentage $(\mathrm{p}=0.005, \mathrm{Cl}: 34.599,193.595)$, cholesterol level $(p=0.008, C l:-39.934,-6.286)$, Triglyceride level $(p=0.04, C l: 0.064$, 56.546), urea level $(\mathrm{p}<0.001, \mathrm{Cl}: 9.356,28.743)$, creatinine ( $\mathrm{p} \leq 0.001, \mathrm{Cl}: 2.345$, $3.960)$, urinary microalbumin $(p=0.024, \mathrm{Cl}: 0.0296,0.399)$, urinary microalbumin/ creatinine ratio $(p=0.006, \mathrm{Cl}: 0.791,4.616)$, and ferritin level $(p=0.025, \mathrm{Cl}: 0.044$, 0.633).

As well, univariate analysis revealed a negative linear relationship between UA level and GFR ( $p<0.001, \mathrm{Cl}:-1.127,-0.486)$

Building a multiple model, including the entire variable with significant association with the UA in the univariate analysis showed that the UA level in RA is determined by GFR, microalbumin creatinine ratio, cholesterol level, monocytes count and DAS score. The adjusted R2 of the model was 54

Conclusions: Whether serum uric acid is merely a marker that reflects the integration of co-morbidities and subclinical renal impairment or a true risk-causative factor for CVD outcome remains as an important question, therefore it is important to know the determinant of UA level and control it.

Disclosure of Interest: None declared

DOI: 10.1136/annrheumdis-2018-eular.4249

\section{$\mathrm{AB} 0404$ \\ APELIN CONCENTRATIONS ARE ASSOCIATED WITH A REDUCED LEFT ATRIAL VOLUME INDEX AND IMPROVED SYSTOLIC FUNCTION IN PATIENTS WITH RHEUMATOID ARTHRITIS}

S. Gunter ${ }^{1}$, C. Robinson ${ }^{1}$, L. Tsang ${ }^{1}$, P.H. Dessein ${ }^{2}$, A.M. Millen ${ }^{1} .{ }^{1}$ Cardiovascular Pathophysiology and Genomics Research Unit, University of Witwatersrand, Johannesburg, South Africa; ${ }^{2}$ Free University Brussels, Brussels, Belgium

Background: We recently reported that apelin concentrations are associated with reduced atherosclerosis and plaque vulnerability as well as improved aortic function in rheumatoid arthritis $(R A)^{1,2}$. These relations were influenced by RA characteristics ${ }^{1,2}$. Besides protecting against atherosclerosis, apelin is also a vasoactive peptide that improves cardiac contractility. In this regard, patients with RA experience a 2 -fold increased risk of developing heart failure ${ }^{3}$. RA patients often demonstrate diastolic dysfunction and heart failure with a preserved ejection fraction (HFpEF). Traditional cardiovascular risk factors do not fully explain the increased heart failure incidence in this population. Metabolic risk factor driven inflammation is highly implicated in HFpEF.

Objectives: This study aimed to determine whether apelin can impact left ventricular function in RA and whether disease characteristics can modify this potential effect.

Methods: Relationships of apelin concentrations with echocardiographically determined markers of systolic and diastolic function including stroke volume, endocardial fractional shortening, midwall fractional shortening, ejection fraction, relative wall thickness, left ventricular mass, mitral inflow $(E / A)$, filling pressure $(E /$ $e^{\prime}$ ) and left atrial volume index ( $\left.L A V I\right)$ were determined in multivariable regression models among 169 patients without established cardiovascular disease.

Results: In demographic characteristic adjusted analysis, rheumatoid factor (RF) positivity, joint deformity counts, and CRP were associated with increased apelin concentrations ( $p=0.01,0.02$ and 0.05 , respectively). Apelin was associated with a reduced $L A V I[\beta(S E)=-4.6(2.2) ; p=0.04]$ but not with $E / A$, lateral $e^{\prime}$ or $E / e$ ( $p>0.05$ for all). RA characteristics including disease duration, CRP, erythrocyte sedimentation rate (ESR), RF positivity, and joint deformity counts did not impact apelin concentration-diastolic function marker relationships (interaction $\mathrm{p}$ values $>0.05$ ). Apelin levels were associated with increased endocardial fractional shortening $[\beta(S E)=5.99$ (2.97); $p=0.04]$ and midwall fractional shortening $[\beta(S E)$ $=6.92(3.0) ; p=0.03]$. The ESR and anti-citrullinated peptide antibody (ACPA) status impacted the apelin level-endocardial fractional shortening relationships (interaction $\mathrm{p}=0.05$ and 0.01 , respectively). In stratified analysis, apelin concentrations were associated with improved endocardial fractional shortening in those with $[\beta(S E)=14.1$ (3.9); $p=0.001$ ] but not without an $E S R>12 \mathrm{~mm} / \mathrm{hr}$ (median value), and in those with $[\beta(S E)=8.2(3.7) ; p=0.03]$ but not without $A C P A$ positivity.

Conclusions: In RA, apelin concentrations are associated with a reduced LAV irrespective of RA activity and severity characteristics. Apelin concentrations are also associated with improved endocardial fractional shortening in patients with RA, particularly in those with high-grade inflammation and ACPA positivity. Whether apelin can improve left ventricular systolic and diastolic function in RA merits further exploration in longitudinal studies.

\section{REFERENCES:}

[1] Gunter S, et al. Atherosclerosis 2017;256-75.81.

[2] Gunter S, et al. Clinical Rheumatology 2018:in press.

[3] Nicola PJ, et al. Arthritis Rheum 2005:52;412-20.

Disclosure of Interest: None declared

DOI: 10.1136/annrheumdis-2018-eular.5124

\section{AB0405 CLINICAL FEATURES AND PROBLEMS OF ELDERLY ONSET RHEUMATOID ARTHRITIS IN ULTRA-AGEING SOCIETY -SINGLE CENTRE RETROSPECTIVE COHORT STUDY-}

T. Kurasawa, Y. Okada, A. Shibata, S. Saito, K. Chino, A. Okuyama, H. Takei, T. Kondo, K. Amano. Department of rheumatology and clinical Immunology, Saitama Medical Center, Saitama Medical University, Kawagoe, Japan

Background: Japan is the ultra-ageing society ahead of any other country in the world, which ageing rate (the ratio of the population aged 65 and older to the total population) was reported to be $27.3 \%$ on October 1,2016 . The rate of aged patients, who followed up at the division of rheumatology in Saitama medical centre, had exceeded $40 \%$.

Objectives: The aim of our study is to reveal recent clinical features and problems of elderly onset rheumatoid arthritis (EORA) patients for better management. Methods: Patients had a diagnosis by 1987 classification criteria or 2010 ACR/ EULAR criteria. We firstly listed up RA patients who were followed up our hospita from April 1 to September 30, and above aged 65 years old as of September 30 Then we retrospectively collected clinical information of EORA patients who onset 